\title{
Neurosurgery in the older patient
}

\author{
IAN STEWART \\ M.R.C.P.
}

\author{
Paul Millac \\ M.D., M.R.C.P.
}

\author{
R. H. SHEPHARD \\ F.R.C.S. \\ Department of Neurology, Leicester Royal Infirmary, and the \\ Regional Neurosurgical Centre, Derbyshire Royal Infirmary
}

\section{Summary}

The outcome of brain and spinal surgery has been assessed in 141 patients who were over the age of 65 at the time of operation. Patients tolerated investigation and surgery remarkably well and good results were obtained for benign lesions. Survival times and the contribution of surgery to reducing disability in malignant disease were disappointing.

\section{Introduction}

Improved diagnostic methods, anaesthetic and operative techniques have extended the range of brain and spinal surgery in recent years into the older age group. Increasingly, the referring physician and neurosurgeon have to consider when to embark upon the investigation of an elderly patient with advancing neurological disability. There is little information to indicate how their patient is likely to fare should he need a major neurosurgical procedure. For this reason we have ascertained the outcome of surgery in all our patients over the age of 65 seen during a 12-year period.

\section{The patients}

These were all investigated in the Department of Neurology at Leicester Royal Infirmary or at the Regional Neurosurgical Centre in Derby between 1959 and 1971. There were 141 patients for whom details were adequate and they have been grouped according to the reason for operation and the pathology demonstrated (Table 1).

\section{Results \\ Craniotomy for benign disorders}

There were nine patients with meningiomas. Two patients, both aged 66, had internal carotid artery aneurysms clipped following subarachnoid haemorrhage. The four remaining operations were for pituitary tumour, intracerebral haematoma, chiasmatic arachnoiditis and section of the pituitary stalk for a diabetic retinopathy.

The follow-up in this group extended from 6 months to 7 years with a mean of 4 years. Eleven patients, including seven with meningiomas and both those with aneurysms, did well with resolution or considerable reduction in their neurological disability. The patients with chiasmatic arachnoiditis and diabetic retinopathy derived modest benefit from operation in common with one patient with an olfactory groove meningioma who had advanced disability before surgery. Three patients with meningiomas required second operations after intervals ranging from 3 to 7 years. A 70-year-old man with a meningioma developed a hemiplegia postoperatively and died of cerebral infarction 3 months later.

\section{Laminectomy for benign disorders}

The diagnoses are shown in Table 2. Follow-up averaged 3 years for the eight men and two women who underwent a cervical laminectomy for spondylosis. Pre-operatively, all had advancing disability sufficient to threaten their independence and signs

TABLE 1. Operations undertaken

\begin{tabular}{lcccc}
\hline & $\begin{array}{c}\text { No. of } \\
\text { patients }\end{array}$ & Males : females & $\begin{array}{c}\text { Mean age } \\
\text { in years }\end{array}$ & $\begin{array}{c}\text { Operative } \\
\text { mortality }\end{array}$ \\
\hline Craniotomy for benign disorders & 15 & $3: 12$ & 68 & 1 \\
Laminectomy for benign disorders & 39 & $21: 18$ & 69 & 0 \\
Craniotomy for malignant disease & 30 & $25: 5$ & 69 & 3 \\
Laminectomy for malignant disease & 20 & $11: 9$ & 69 & 1 \\
Operations for relief of pain & 16 & $7: 9$ & 70 & 0 \\
Burr holes-subdural & 14 & $9: 5$ & 72 & 1 \\
Burr hole biopsy & 7 & $6: 1$ & 70 & 1 \\
Totals & 141 & $82: 59$ & & $7(5 \%)$ \\
\hline
\end{tabular}


TABLE 2. Laminectomy for benign conditions

\begin{tabular}{lr}
\hline Cervical Spondylosis & 10 \\
Lumbar Disc Disease & 12 \\
Dorsal Meningiomas & 5 \\
Neurofibromas & 7 \\
Angiomas & 2 \\
Paget's & 1 \\
Tuberculosis & 1 \\
Arachnoiditis & 1 \\
& 39 \\
\hline
\end{tabular}

of cord compression which was confirmed by myelography. Afterwards three were judged much improved, three derived moderate benefit and in the other four there was little change. One patient died of an unrelated cause within 6 months of surgery.

Eight patients with lumbar disc disease were considerably improved by operation, two moderately and symptoms persisted unchanged in two patients.

All those with benign spinal tumours were improved by surgery. One patient died 1 year later of a coincidental illness and one neurofibroma recurred after 5 years.

Two patients with angiomas in the dorsal region underwent surgery for increasing weakness of the legs. A 72-year-old female who had been deteriorating for more than 3 years before operation improved steadily afterwards and was alive with much less disability 4 years later. A 68-year-old man showed only slight improvement and died 4 months postoperatively with a urinary infection and pressure sores.

A man with Paget's disease and a paraparesis was symptom-free 9 months after decompression. A 66year-old woman with back pain of 15 months' duration, paraparesis for a year and incontinence of urine for 4 months had a dorsal extradural mass explored which was histologically shown to be tuberculous. Gradual improvement with immobilization and antituberculous chemotherapy was recorded up to 18 months later. The last patient with cord disease was found to have arachnoiditis which was not improved after exploration.

\section{Craniotomy for malignant disease}

Four patients had a previous history of malignant disease (colon three, larynx one) and all proved to have metastases. Four others without any previous history of neoplasia were also found to have metastases (bronchus three, one unidentified).

Twenty patients had astrocytomas. Twelve were graded histologically III or IV, four grade II and four were unclassified. The remaining two patients had an orbital myeloma and malignant polypi extending from the nasal sinuses.

Three patients died within $24 \mathrm{hr}$ of surgery, all of whom were in poor condition beforehand. Survival times were known for twenty-two patients. Apart from the patient with malignant polypi, who survived 16 months, only a woman with a grade III astrocytoma lived for more than a year. She alone received radiotherapy. Eighteen of the twenty-two patients lived 5 months or less, and only four were significantly improved by operation. Within this small number of patients, the outcome was not influenced by whether the tumour was primary or secondary nor by the histological grading.

\section{Laminectomy for malignant disease}

Operation was not offered to patients with a complete spinal lesion in the presence of known malignant disease or bony destruction on spinal X-rays. Surgery was undertaken to establish the diagnosis and preserve function not yet lost. Twelve patients had metastases, three from bronchogenic carcinomas and one each from breast, prostate and kidney; in the remaining six the site of the primary was not known. Three more patients had sarcomas, three had lymphomas, one a chordoma and from one a neurofibroma with malignant change was removed.

One woman of 72 with a lymphoma did outstandingly well having presented with a 2-month history of back pain and weakness of the legs with retention of urine for 2 weeks. After surgery and radiotherapy her neurological disability almost completely resolved and she continued well until her death 5 years later from a cardiac complaint. In the 66-year-old patient with the chordoma, the tumour was partially removed and after radiotherapy the patient experienced moderate amelioration of symptoms which lasted for 8 years. A woman of 70 had a sarcomatous tumour decompressed and with radiotherapy remained mobile for 3 years despite metastases elsewhere. Two other patients were improved for up to 6 months. There was one operative death.

The remaining fourteen patients were not helped by surgery and seven survived a month or less. Four of them were given radiotherapy post-operatively with little discernible effect.

\section{Operations for relief of pain}

Four patients underwent a posterior fossa craniectomy with division of the sensory root of the fifth nerve for trigeminal neuralgia which had not responded to carbamazepine. In one instance the ninth nerve was also divided as the patient was considered to have glossopharyngeal neuralgia as well. Three of the four were relieved of pain completely, but the last relapsed 2 months after operation.

A 72-year-old man with glossopharyngeal neuralgia alone had the ninth nerve divided through a posterior fossa craniectomy. Afterwards he was pain-free but 
developed abdominal symptoms and died 2 months later without the cause of death being ascertained.

An hypophysectomy for bone pains complicating breast cancer was carried out in a 67-year-old woman who had already undergone bilateral oophorectomy without relief. Only temporary benefit resulted and she died 4 months later. Spinothalamic tractotomies were performed in five patients with malignant disease and in another with sciatica and an abdominal mass. All patients were cured of their pain in the short term but longer follow-up was lacking. An attempt to relieve longstanding discomfort in an amputation stump by tractotomy was unrewarding.

Three patients had a hemilaminectomy and posterior rhizotomy for limb pain. One patient with lumbar disc disease and another with breast carcinoma were helped by surgery. The third patient who had post-herpetic neuralgia did not benefit.

\section{Burr holes}

Seven burr hole biopsies were taken from ill patients thought to have malignant disease. One was unsuccessful, but the others yielded the definitive diagnosis. One patient died shortly after the procedure, the remainder survived between 10 days and 8 weeks before dying of the primary disease.

Fourteen patients were suspected of having subdural haematomas and had burr hole explorations. Haematomas were evacuated from six patients with good recovery in five of them. The sixth patient died immediately after tapping and was shown at autopsy to have an associated area of infarction. The eight remaining patients turned out to have a variety of disorders but were not harmed by the procedure.

\section{Discussion}

When Pennybacker (1968) compared the neurosurgical admissions of patients aged 65 or older to the Radcliffe Infirmary, Oxford, in 1967 he found a fourfold increase over the corresponding figures for 1938. There can be little doubt of this continuing trend which will be enhanced by the increasing availability of safe and effective screening tests such as echo-encephalography and isotope scanning. The patients in our series with meningiomas comprised $9 \%$ and those with malignant cerebral tumours $6 \%$ of the total operated upon for these conditions during the period under review.

There are few studies of mortality amongst older patients undergoing neurosurgery. Kloss and Scharfetter (1968) quote a $40 \%$ mortality amongst seventy-one patients over the age of 60 years who were operated upon for cerebral tumours. Stellar and Cooper (1970) indicated a doubling of the mortality and serious morbidity associated with cryothalamotomy in the seventh and eighth decades. However, the arbitrary definition of operative mortality as death within a month of surgery conveys a false impression of the outcome of intervention in patients, many of whom had rapidly advancing lethal nervous system diseases. By assessing cases individually, our study shows that the contribution of surgery to demise was remarkably low at $5 \%$.

It plainly emerges that operations for benign tumours of the brain and spine in the elderly were unquestionably worthwhile and tolerated well together with the neuroradiological diagnostic procedures leading up to surgery. It is relevant to consider that the life expectancy of a 70-year-old female is of the order of 13 years and that of a male 10 years.

Operations for relief of pain were generally successful and in the more controversial areas of cervical spondylosis and back pain, surgery achieved much as might be anticipated in a younger age group. The operations for causalgia in an amputation stump and for post-herpetic neuralgia were performed early in the period under review. Many patients with these conditions in succeeding years have been spared such unrewarding procedures.

On the other hand, the attempt to improve the quality of survival in older patients with malignant disease was disappointing. Unfortunately, operation may be the only way of establishing the diagnosis with certainty and occasional patients with malignancy, well demonstrated by one patient with a lymphoma, may be helped greatly. Our experience would confirm the view that there is little to be gained by performing a laminectomy in most instances where there is a proven primary growth, bony destruction on plain films of the spine and advanced neurological disability. Where possible, operation should be deferred whilst suspicious chest X-ray findings are pursued. The isotope brain scan has proved more helpful than other investigations in detecting multiple intracranial metastases. It may be reasonable to delay surgery where investigations point to malignant cerebral neoplasms and where there is a good response to dexamethasone since relief may be sustained for several months with acceptable side effects.

In some units burr hole biopsy is seldom undertaken nowadays because of the risk of uncontrollable bleeding within a vascular tumour. Nevertheless, it remains a useful diagnostic technique in certain situations.

In common with Pennybacker (1968), we noticed the paucity of referrals for exclusion of subdural haematoma. This may be because such patients are dealt with by general surgical firms or because angiography has been used to exclude the diagnosis. However, 
we suspect subdural haematomas are more likely to be overlooked in the elderly.

We could not estimate the duration of hospitalization for our patients to compare with younger age groups because of transfers to other hospitals for convalescence. It was our impression that postoperative recovery in the older patient was not significantly delayed.

\section{References}

KLoss, K. \& Scharfetter, F. (1968) Neurosurgery in the older patient. Landarzt, 44, 1251.

Pennybacker, J. (1968) Neurosurgery in the older patient. Ceskoslovenská neurologie, 31, 73.

STELLAR, S. \& Cooper, L.S. (1970) Neurosurgery in the older patient. Journal of the American Medical Association,

$18,845$. 